\title{
Applicative Study (Part I): The Excellent Conditions to Remove in Batch Direct Textile Dyes (Direct Red, Direct Blue and Direct Yellow) from Aqueous Solutions by Adsorption Processes on Low-Cost Chitosan Films under Different Conditions
}

\author{
Vito Rizzi ${ }^{1}$, Alessandra Longo ${ }^{1}$, Paola Fini' ${ }^{2}$, Paola Semeraro ${ }^{1}$, Pinalysa Cosma ${ }^{1,2}$, \\ Esther Franco ${ }^{3}$, Rocío García ${ }^{3}$, Marcela Ferrándiz ${ }^{3}$, Estrella Núñez ${ }^{4}$, \\ José Antonio Gabaldón' ${ }^{4}$ Isabel Fortea ${ }^{4}$, Enrique Pérez ${ }^{5}$, Miguel Ferrándiz ${ }^{5}$ \\ ${ }^{1}$ Università degli Studi “Aldo Moro” di Bari, Dip. Chimica, Bari, Italy \\ ${ }^{2}$ Consiglio Nazionale delle Ricerche CNR-IPCF, UOS Bari, Bari, Italy \\ ${ }^{3}$ Biotechnology Department, Textile Industry Research Association (AITEX), Alcoy, Spain \\ ${ }^{4}$ Departamento Ciencia y Tecnología de Alimentos, Universidad Católica San Antonio de Murcia, Guadalupe, \\ Murcia, Spain \\ ${ }^{5}$ Colorprint Fashion, SL, Avda. Fco. Vitoria Laporta, Muro de Alcoy (Alicante), Spain \\ Email: pinalysa.cosma@uniba.it, vito.rizzi@uniba.it, p.fini@ba.ipcf.cnr.it, paola.semeraro@uniba.it, \\ longo.alessandra88@gmail.com, enunez@pdi.ucam.edu, jagabaldon@ucam.edu, mifortea@ucam.edu, \\ direccion@colorprintfashion.com, produccion@colorprintfashion.com
}

Received 10 June 2014; revised 10 July 2014; accepted 4 August 2014

Copyright (C) 2014 by authors and Scientific Research Publishing Inc.

This work is licensed under the Creative Commons Attribution International License (CC BY).

http://creativecommons.org/licenses/by/4.0/

(c) (i) Open Access

\begin{abstract}
In recent years the development of chitosan $(\mathrm{CH})$ based materials as useful adsorbent polymeric matrices is an expanding field in the area of adsorption science. Even though $\mathrm{CH}$ has been successfully used for dye removal from aqueous solutions due to its low cost, no considerations have been made about, for example, the effect of changing the $\mathrm{pH}$ of chitosan hydrogelor about the dehydrating effect of Ethanol (EtOH) treatment of chitosan film on the dyes removal from water. Consequently in our laboratory we carried out a study focusing the attention, mainly, on the potential use of $\mathrm{CH}$ films under different conditions, such as reducing the intrinsic $\mathrm{pH}$, increasing the hydrophobic character by means of ethanol treatment and neutralization of $\mathrm{CH}$ films to improve their absorption power. Textile anionic dyes named Direct Red 83:1, Direct Yellow 86 and Direct

How to cite this paper: Rizzi, V., et al. (2014) Applicative Study (Part I): The Excellent Conditions to Remove in Batch Direct Textile Dyes (Direct Red, Direct Blue and Direct Yellow) from Aqueous Solutions by Adsorption Processes on Low-Cost Chitosan Films under Different Conditions. Advances in Chemical Engineering and Science, 4, 454-469.

http://dx.doi.org/10.4236/aces.2014.44048
\end{abstract}


Blue 78 have been studied with the aim of reducing the contact time of $\mathrm{CH}$ film in waste water improving the bleaching efficiency. Neutralized acid $\mathrm{CH}$ film and longtime dehydrated one result to be the better films in dye removal from water. Also the reduction of the $\mathrm{CH}$ solution acidity during the film preparation determines the decreasing of the contact time improving the results. The effect of initial dye concentration has been examined and the amount of dye adsorption in function of time $t, q_{t}\left(\mathrm{mg} / \mathrm{cm}^{2}\right)$, for each analyzed film has been evaluated comparing the long term effect with the decoloration rate. A linear form of pseudo-first-order Lagergren model has been used and described. The best condition for removing all examined dyes from various dye solutions appears to be the dehydration of a novel projected $\mathrm{CH}$ film obtained by means of the film immersion in EtOH for 4 days. Also $\mathrm{CH}$ films prepared by well-known literature procedure and neutralized with $\mathrm{NaOH}$ treatment appear having an excellent behavior, however the film treatment requires a large quantity of water and time.

\section{Keywords}

Chitosan Film, Textile Dyes, Adsorption Processes, Wastewater

\section{Introduction}

\subsection{Dyes and Wastewater Treatments}

Water represents the most very important human resource with economic, social, political and environmental importance throughout the world [1]. Recently, different pollutants are inflowing aquatic systems since the rapid industrialization and urbanization [2]. Considerable volumes of water were consumed by textile, paper, plastics and dyestuffs industries due to the use of very different chemical reagents during manufacturing and coloring of their products. Subsequently, they produce a considerable amount of polluted wastewater and among them color is the first contaminant to be recognized in wastewater and the presence of very small amounts of dyes in water is highly visible and undesirable [3]. Thus, every year, tons of dyes are discharged from textile, pharmaceutical, packed food, pulp and paper, paint, plastics, petroleum, electroplating, and cosmetics industries into the environment worldwide [4]. This together with a strict legislation on the discharge of these toxic products in the environment makes necessary to develop various efficient technologies for the removal of pollutants from wastewater [5] with the result that the treatment of wastewater becomes a matter of dominant importance. Efforts to develop processes for removing assorted pollutants have mainly attempted by researchers in the analytical, environmental and material sciences and more recently different technologies and processes are used. Extensively used technology for the removal of both inorganic and organic material could be classified in biological treatments, membrane processes, advanced oxidation processes, chemical and electrochemical techniques [6], and adsorption procedures [7]. In spite of the availability of different proposed treatments, the physical adsorption by means of sorbent materials represents one of the most fashionable methods since proper design of the adsorption process will produce high-quality treated effluents. Physical adsorption is a well-known equilibrium separation process and since it is widely recognized that adsorption using low-cost adsorbents is an useful and economic method for wastewater decontamination, a number of materials have been extensively investigated [5].

\subsection{Chitosan: A Low Cost Dyes-Bioadsorbent}

Recently the attention has been focused on a variety of biosorbent materials [8] [9]. Due to their low cost the use of adsorbents composed of natural polymers has attracted significant interest, and polysaccharides such as Chitosan $(\mathrm{CH})$ and its derivatives have received great attention. $\mathrm{CH}$ is an effective adsorbent compared with activated carbons and other common adsorbents used in treatment of organic or inorganic contaminated water [10]. It is a derivative of chitin, one of the most abundant natural polymers in the biosphere, that represent the main component of the exoskeletons of marine crustaceans (e.g., shrimps, crabs, krill), which are available in large amounts as a byproduct of food processing [11]. Depending on the source and preparation procedure, the number of glucosamine residues (denoted as the degree of deacetylation) on the linear polysaccharidic chain of $\mathrm{CH}$ 
is above $60 \%$ [12]. $\mathrm{CH}$ is considered a super high-capacity adsorbent for contaminant removal from water due to its ability of binding contaminants through hydroxyl and amino groups on the surface [13]. In particular due to the protonation of the primary amino groups of $\mathrm{CH}$ by acidic media, it can strongly adsorb anionic dyes such as acid, reactive and direct dyes, by electrostatic attraction [14] [15] and also metal anions by ion exchange. Since $\mathrm{pH}$ variation leads to the difference in the degree of ionization of the adsorptive molecule and the surface properties of adsorbent, the $\mathrm{pH}$ of the medium is one of the most important factors affecting the capacity of adsorbent in wastewater treatment and consequently the efficiency of adsorption is strictly related on the media $\mathrm{pH}$ [16]. It is worth noting that the amount of dye removal by means of adsorption processes is greatly related to the chemical nature [17] (For more details see Ref. [18]) and to the initial concentration of the dyes. Concerning this aspect several ways for classification of commercial dyes are known in literature and generally they can be classified in terms of chemical structure, color and application method [19]. However, due to the complexities of the color nomenclature from the chemical structure system, the classification based on application is often considered more useful [20]. A classification based on their particle charge upon dissolution in aqueous application medium such as cationic (all basic dyes), anionic (direct, acid, and reactive dyes), and non-ionic (dispersed dyes) has been used yet [18]. Since the increasing number of review articles published on the use of $\mathrm{CH}$ and its derivatives as adsorbents for the removal of contaminants from wastewaters [6] in the present paper we summarize the ability of modified $\mathrm{CH}$ films in binding different textile dyes, in particular azoic ones, considered extremely toxic due to the presence of toxic amines as by-products in the effluent under varying conditions [18] [21]-[23]. It has been shown the modified procedure to prepare $\mathrm{CH}$ films and the sorption mechanism related to the dye decoloration rate. The attention has been focused on three direct dyes, Direct Red 83:1 (DR), Direct Yellow 86 (DY) and Direct Blue 78 (DB), analyzing the $\mathrm{pH}$ effect both of the medium and of the film on dye uptake from aqueous solutions. Also the influence of neutralization and increasing hydrophobicity of $\mathrm{CH}$ films has been examined. The obtained results indicate that this novel modality of producing $\mathrm{CH}$ films allows the treatment of a large amount of wastewater which can be purified with an efficiency around of $100 \%$ in a very short period of time using less energy and low cost procedure.

\section{Material and Methods}

All the chemicals used were of analytical grade and samples were prepared using double distilled water. Commercial grade $\mathrm{CH}$ powder (from crab shells, highly viscous, with deacetylation degree $\geq 75 \%$ ), Acetic acid (99.9\%), Ethanol (EtOH) (99.9\%) and glycerol (+99.5\%) were purchased from Sigma-Aldrich. Direct Red 83:1, Direct Yellow 86 and Direct Blue 78 were received by Colorprint Fashion, S.L within the LIFE+ European Project named "DYES4EVER" (Demonstration of Cyclodextrin Techniques in Treatment of Waste Water in Textile Industry to Recover and Reuse Textile Dyes) and used without further purification. Direct dyes characteristics are: Direct Red (Color Index Number: 83:1), chemical formula: $\mathrm{C}_{33} \mathrm{H}_{20} \mathrm{~N}_{6} \mathrm{Na}_{4} \mathrm{O}_{17} \mathrm{~S}_{4}$, MW: $992.77 \mathrm{~g}$ mol $^{-1}$; Direct Yellow (Color Index Number: 86), chemical formula: $\mathrm{C}_{39} \mathrm{H}_{30} \mathrm{~N}_{10} \mathrm{Na}_{4} \mathrm{O}_{13} \mathrm{~S}_{4}$, MW: $1066.94 \mathrm{~g} \mathrm{~mol}^{-1}$ and Direct Blue (Color Index Number: 78), chemical formula: $\mathrm{C}_{42} \mathrm{H}_{25} \mathrm{~N}_{7} \mathrm{Na}_{4} \mathrm{O}_{13} \mathrm{~S}_{4}$, MW: $1055.1 \mathrm{~g} \cdot \mathrm{mol}^{-1}$, (see Scheme 1).

Dye stock solutions with a concentration of $10^{-4} \mathrm{M}$ were prepared and dilutions were carried out with double distilled water to obtain different concentrations, $5 \times 10^{-5} \mathrm{M}$ and $5 \times 10^{-6} \mathrm{M}$.

The $\mathrm{pH}$ of the various aqueous solutions has been adjusted using concentrated $\mathrm{HCl}$ and $\mathrm{NaOH}$ solutions.

UV-Vis absorption spectra were recorded in the $200-800 \mathrm{~nm}$ range, at a $0.5 \mathrm{~nm} / \mathrm{s}$ scan rate, using a Shimadzu UV-Vis spectrophotometer mod. 1601.

\subsection{Preparation of Chitosan Films}

The standard procedure used in literature to prepare CH films [24] [25] was adopted. In order to optimize the adsorption processes, $\mathrm{pH}$ medium effect and dehydration of $\mathrm{CH}$ films by means of $\mathrm{EtOH}$ were studied, too. $\mathrm{CH}$ powder was dissolved in $0.8 \%(\mathrm{v} / \mathrm{v})$ aqueous acetic acid solution in order to obtain a $1 \%(\mathrm{w} / \mathrm{v})$ chitosan concentration by constant continuous stirring for $24 \mathrm{hrs}$. $200 \mu \mathrm{L}$ of glycerol were added every $100 \mathrm{~mL}$ of CH acetic solution. Then the solution was filtered through a coarse sintered glass filter and degassed for $1 \mathrm{hr}$. After degassing, the $\mathrm{CH}$ solution was poured into a plastic Petri plate. This plate was maintained in an oven at $60^{\circ} \mathrm{C}$ for $24 \mathrm{hrs}$. A thin $\mathrm{CH}$ membrane was obtained. 


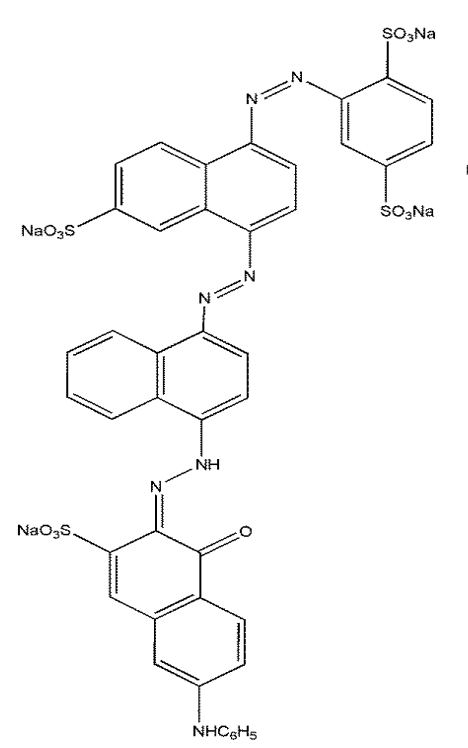

(a)

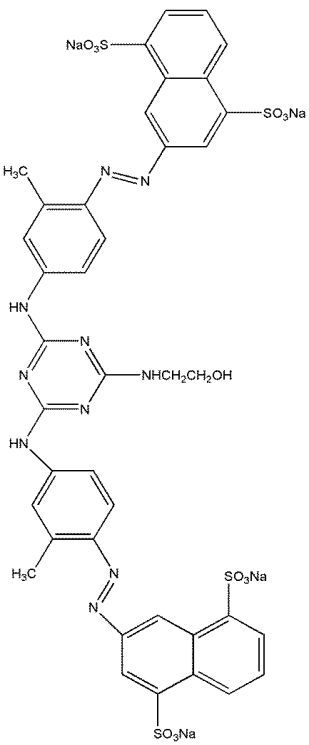

(b)

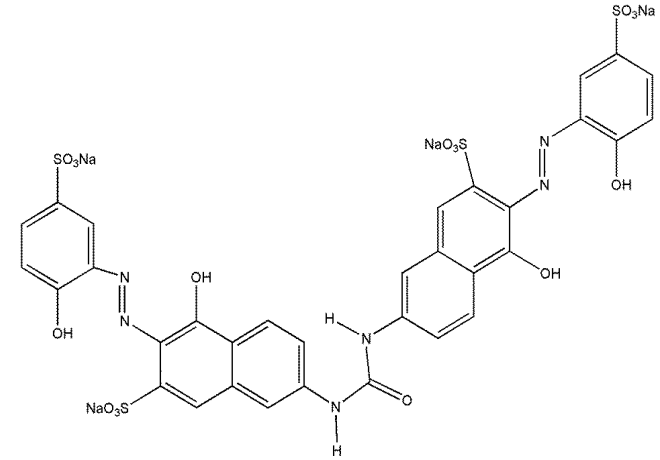

(c)

Scheme 1. Molecular structure of used dyes: Direct Blue 78 (a); Direct Yellow 86 (b) and Direct Red 83:1 (c).

In order to reduce the intrinsic acidity of $\mathrm{CH}$ hydrogel medium and to low the total cost to prepare $\mathrm{CH}$ films, a novel procedure was adopted modifying the standard procedure known in literature. In particular an excess of $\mathrm{CH}$ powder was used. $2 \mathrm{~g}$ were solubilized in $100 \mathrm{~mL}$ of known acetic acid solution $(0.1 \% \mathrm{v} / \mathrm{v})$ containing the same amount of glycerol ( $200 \mu \mathrm{L}$ every $100 \mathrm{~mL}$ acetic solution). In this way the $\mathrm{CH}$ solution was almost completely neutralized and $\mathrm{pH} 6.2$ was established. As indicated in the previous procedure, the solution was filtered, however in this case a great amount of $\mathrm{CH}$ powder remains insoluble, consequently could be recovered and potentially used to prepare new films reducing the total cost. Small amount of $\mathrm{CH}$ was required to realize the membrane. The obtained free standing films were sliced in order to obtain $2.5 \mathrm{~cm} \times 2.5 \mathrm{~cm}$ films.

About the $\mathrm{CH}$ films obtained according to the standard procedure illustrated in Krajewska et al. [24] [25], the effect of film neutralization with a $\mathrm{NaOH}$ solution $(0.2 \mathrm{M})$ and the effect of the dye solutions $\mathrm{pH}$ on dye adsorption processes, were studied too. Concentrated $\mathrm{NaOH}$ and $\mathrm{HCl}$ were employed to adjust the $\mathrm{pH}$ of dye solutions, establishing $\mathrm{pH} 12$ and 2, respectively.

Neutralization of acid CH films was obtained immerging the film in the $0.2 \mathrm{M} \mathrm{NaOH}$ solution $(40 \mathrm{~mL})$ for 30 min. Successively the film was washed by immersion in double distilled water for six days changing water every day to ensure the complete neutralization. The effect of EtOH treatment was estimated immerging prepared $\mathrm{CH}$ films in $\mathrm{EtOH}(40 \mathrm{~mL})$ for $24 \mathrm{~h}$ and $96 \mathrm{~h}$.

\subsection{Batch Experiments and Adsorption Isotherms}

Adsorption kinetics were carried out by in batch technique. Batch experiments were carried out for determining the adsorption isotherms of dyes onto the various adsorbent $\mathrm{CH}$ films in a glass beaker. The dye aqueous solutions were magnetically stirred at a constant rate $(140 \mathrm{rpm})$, allowing sufficient time for reaching adsorption equilibrium. It was assumed that the applied stirring speed allows all the film surface area to come in contact with dye molecules over the course of the experiments. In each experiment a fixed volume $(40 \mathrm{~mL})$ of dye aqueous solution at constant dye concentration $\left(5 \times 10^{-5} \mathrm{M}\right.$ and $\left.5 \times 10^{-6} \mathrm{M}\right)$ was used. The study was performed at room temperature to be representative of environmentally relevant condition. All experiments were carried out in duplicate and the average value was used for further calculation.

For the adsorption kinetic study, sample aliquots were withdrawn from the flask at predetermined time intervals and the UV-Vis absorption spectrum was recorded. Measurements were carried out for several days until the equilibrium was reached, however to estimate the adsorption rate constants only data collect in the first 250 min were employed. According to the procedure used in Run Fang et al. [26], it is possible to evaluate the efficiency on equilibrium of adsorption process using the following decoloration rate equation (Equation (1)): 


$$
E=\frac{A_{0}-A}{A_{0}} \times 100
$$

where $A_{0}$ and $A$ represent the intensity of the dye absorption band at a fixed wavelength, at time zero and at equilibrium time, respectively. The amount of adsorbed dye at a certain time $t, q_{t}\left(\mathrm{mg} / \mathrm{cm}^{2}\right)$ was calculated by Equation (2):

$$
q(t)=\frac{C_{0}-C_{t}}{A_{\mathrm{CH}}} \times V
$$

where $V$ represents the total volume of solution (herein $40 \mathrm{~mL}$ ), $A_{\mathrm{CH}}$ is the area of the analyzed $\mathrm{CH}$ films (obtained redoubling the geometrical area of the films), $C_{0}$ and $C_{t}$ represent the initial concentration and the concentration at time $\mathrm{t}$ for each dye. The $A_{\mathrm{CH}}$ was calculated by the following formula (Equation (3)):

$$
A_{\mathrm{CH}}=2(d \times d)
$$

in which $d$ represent the size of the film side. In this case the film side measures about $2.5 \mathrm{~cm}$, then the total superficial area is about $12.5 \mathrm{~cm}^{2}$. To calculate the mass of adsorbed dye in milligrams, the epsilon values used are reported in Table 1, expressed in $\mathrm{M}^{-1} \cdot \mathrm{cm}^{-1}$ units.

The time evolution of the dye adsorption was interpreted by means of a pseudo-first-order model described by Lagergren in the form:

\begin{tabular}{|c|c|c|c|c|c|c|c|c|}
\hline \multirow{2}{*}{ Film } & \multirow{2}{*}{$\begin{array}{c}\mathrm{q}_{\mathrm{e}, \mathrm{th}} \\
\left(\mathrm{mg} / \mathrm{cm}^{2}\right)\end{array}$} & \multirow{2}{*}{$\begin{array}{c}\mathrm{q}_{\mathrm{e}, \exp } \\
\left(\mathrm{mg} / \mathrm{cm}^{2}\right)\end{array}$} & \multicolumn{3}{|c|}{ Pseudo-first order } & \multicolumn{3}{|c|}{ Pseudo-second order } \\
\hline & & & $\begin{array}{c}\mathrm{q}_{\mathrm{e}, \mathrm{calc}} \\
\left(\mathrm{mg} / \mathrm{cm}^{2}\right)\end{array}$ & $\mathrm{K}\left(\min ^{-1}\right)$ & $\mathrm{R}^{2}$ & $\begin{array}{c}\mathrm{q}_{\mathrm{e}, \mathrm{calc}} \\
\left(\mathrm{mg} / \mathrm{cm}^{2}\right)\end{array}$ & K & $\mathrm{R}^{2}$ \\
\hline \multicolumn{9}{|c|}{ DY/CH new } \\
\hline \multicolumn{9}{|c|}{ Concentrated dye } \\
\hline $\mathrm{CH}$ & 0.17 & 0.16 & 0.14 & $(35.00 \pm 2.00) \times 10^{-4}$ & 0.96 & 0.12 & $(1.32 \pm 1.00)$ & 0.99 \\
\hline $\mathrm{CH} / \mathrm{EtOH} 1 \mathrm{~d}$ & 0.17 & 0.17 & 0.16 & $(9.00 \pm 1.00) \times 10^{-4}$ & 0.95 & 0.07 & $(1.22 \pm 1.00)$ & 0.99 \\
\hline $\mathrm{CH} / \mathrm{EtOH} 4 \mathrm{~d}$ & 0.17 & 0.16 & 0.16 & $(3.00 \pm 1.5) \times 10^{-4}$ & 0.69 & 0.022 & I & I \\
\hline \multicolumn{9}{|c|}{ Diluted dye } \\
\hline $\mathrm{CH}$ & 0.019 & 0.014 & 0.015 & $(20.00 \pm 1.00) \times 10^{-4}$ & 0.97 & 3.7 & l & I \\
\hline CH/EtOH 1d & 0.019 & 0.016 & 0.014 & $(43.00 \pm 4.00) \times 10^{-4}$ & 0.90 & 0.024 & $(81.32 \pm 56.35) \times 10^{-2}$ & 0.89 \\
\hline $\mathrm{CH} / \mathrm{EtOH} 4 \mathrm{~d}$ & 0.019 & 0.016 & 0.012 & $(109.00 \pm 7.00) \times 10^{-4}$ & 0.94 & 0.029 & $1.01 \pm 0.45$ & 0.89 \\
\hline \multicolumn{9}{|c|}{ DY/CH std } \\
\hline \multicolumn{9}{|c|}{ Concentrated Dye } \\
\hline $\mathrm{CH}$ pH12 & 0.17 & 0.17 & 0.12 & $(22.00 \pm 1.00) \times 10^{-4}$ & 0.98 & 0.09 & $(0.14 \pm 0.08)$ & 0.98 \\
\hline $\mathrm{CH} / \mathrm{NaOH}$ & 0.19 & 0.24 & 0.24 & $(40.00 \pm 1.00) \times 10^{-4}$ & 0.99 & 0.38 & $(7.00 \pm 2.00) \times 10^{-3}$ & 0.90 \\
\hline $\begin{array}{c}\mathrm{CH} / \mathrm{EtOH} \\
1 \mathrm{~d}\end{array}$ & 0.19 & 0.17 & 0.26 & $(42.00 \pm 1.00) \times 10^{-4}$ & 0.99 & 0.15 & $(53.09 \pm 14.88) \times 10^{-3}$ & 0.98 \\
\hline $\begin{array}{c}\mathrm{CH} / \mathrm{EtOH} \\
4 \mathrm{~d}\end{array}$ & 0.20 & 0.21 & 0.20 & $(20.00 \pm 1.00) \times 10^{-4}$ & 0.98 & 0.13 & $(62.28 \pm 25.00) \times 10^{-3}$ & 0.98 \\
\hline \multicolumn{9}{|c|}{ Diluted dye } \\
\hline $\mathrm{CH}$ pH 12 & 0.019 & 0.019 & 0.018 & $(24.00 \pm 1.50) \times 10^{-4}$ & 0.97 & 0.0013 & $(62.10 \pm 18.40) \times 10^{-2}$ & 0.99 \\
\hline $\mathrm{CH} / \mathrm{NaOH}$ & 0.020 & 0.026 & 0.025 & $(81.00 \pm 1.00) \times 10^{-4}$ & 0.99 & 0.0036 & $(19.61 \pm 4.66) \times 10^{-2}$ & 0.99 \\
\hline $\begin{array}{c}\mathrm{CH} / \mathrm{EtOH} \\
4 \mathrm{~d}\end{array}$ & 0.020 & 0.022 & 0.0021 & $(50.00 \pm 1.50) \times 10^{-4}$ & 0.99 & 0.023 & $(35.65 \pm 15.04) \times 10^{-2}$ & 0.94 \\
\hline
\end{tabular}

Table 1. Comparison of the pseudo-first-order, pseudo-second-order adsorption rate constants and calculated and experimental $\mathrm{q}_{\mathrm{e}}$ values obtained at different Direct Yellow 86 concentrations. 


$$
\log \left(q_{e}-q_{t}\right)=\log q_{e}-\frac{K t}{2.303}
$$

where $q_{e}$ and $q_{t}$ are the adsorption capacity $\left(\mathrm{mg} / \mathrm{cm}^{2}\right)$ at equilibrium and at time $t(\mathrm{~min})$, respectively; $k\left(\mathrm{~min}^{-1}\right)$ is the adsorption rate constant of pseudo-first-order and was calculated from the linear plot of $\log \left(q_{e}-q_{t}\right)$ versus $\mathrm{t}$ [18].

\section{Results and Discussion}

The UV-Vis absorption spectra of all studied dyes recorded in aqueous solution at different pHs are reported in Figure 1. The absorption spectra are dominated by the classical absorption bands relative to the characteristics chromophore for each dye, result of the interaction between azo functionality $(-\mathrm{N}=\mathrm{N}-)$ and attached aromatic moieties. In general there are weaker bands in the UV region attributable to the electronic transitions related to the aromatic rings, while in the visible range there are more intense and wider bands due to $\pi \rightarrow \pi^{*}$ transitions of do nor groups, e.g. an aromatic nucleus containing an auxochromic group such as alkyl side chains, secondary amine, or OH group [27]-[29]. The characteristic absorption bands in aqueous solution at pH7 are located at 605

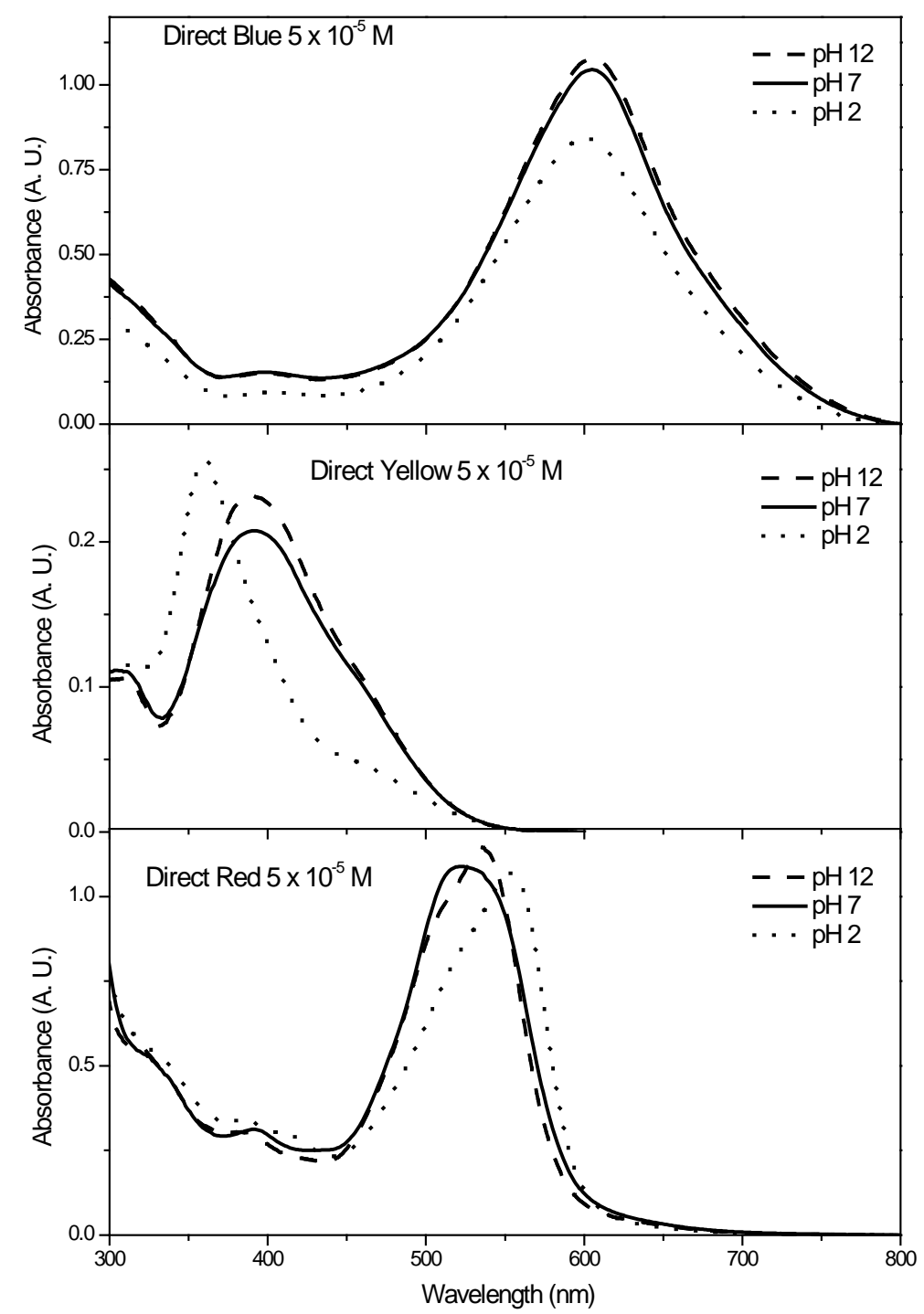

Figure 1. UV-VIS absorption spectra of aqueous solutions of dyes at different $\mathrm{pH}$. 
$\mathrm{nm}$ for DB, at $365 \mathrm{~nm}$ for DY and at $527 \mathrm{~nm}$ for DR. The peak position changes varying the $\mathrm{pH}$ of aqueous solution only for DY and DR, while results to be unaffected by changing solution $\mathrm{pH}$ in the case of DB. In particular for acid and basic $\mathrm{pH}$ the DY absorption band shifts to $390 \mathrm{~nm}$, whereas the DR absorption band at acid $\mathrm{pH}$ shifts to $553 \mathrm{~nm}$ and for basic $\mathrm{pH}$ is located at $535 \mathrm{~nm}$. The decoloration rate has been evaluated monitoring the changes in time of the dye characteristic absorption bands in the visible range spectrum varying the $\mathrm{CH}$ film type and the dye solution properties. In general, for all examined cases, the efficiency of dye adsorption depends on the $\mathrm{pH}$ of the aqueous solution in which the $\mathrm{CH}$ film is immersed influencing not only the surface charge of the adsorbent (due to the presence of functional group such as $\mathrm{NH}_{3}{ }^{+}$group on $\mathrm{CH}$ film surface), the degree of ionization of the material present in the solution and the dissociation of functional groups on the active sites of the adsorbent, but also the solution dye chemistry [14] [18].

In Figures 2 (panel a-f) the comparison between standard $\mathrm{CH}$ film (CH std), prepared following the classical
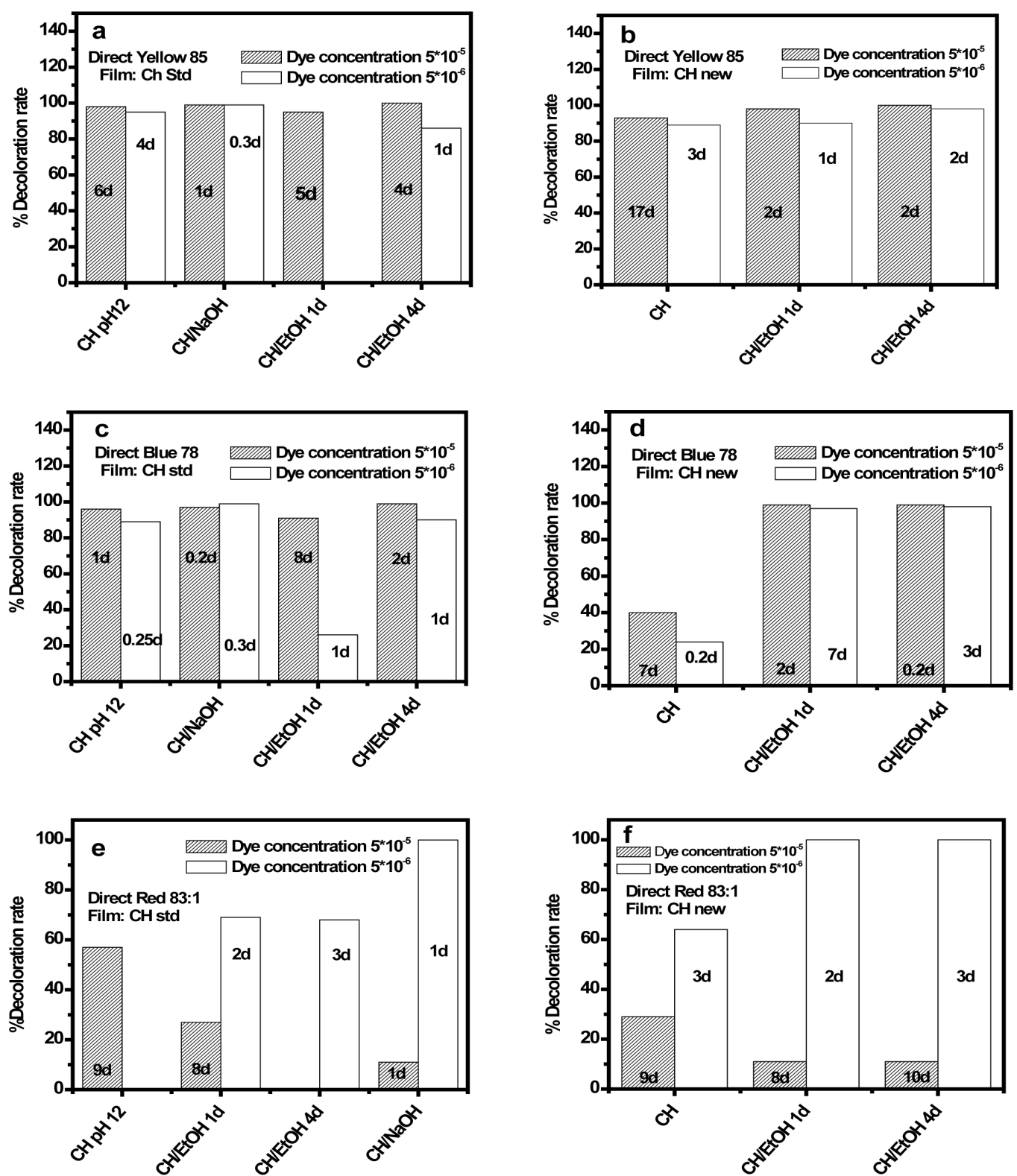

Figure 2. Comparison of decoloration rate percentage for different dyes in presence of CH films. (a) DY/CH std; (b) DY/CH new; (c) DB/CH std; (d) DB/CH new; (e) DR/CH std; (f) DR/CH new. Dense pattern is referred to highest dye concentration 5 $\times 10^{-5} \mathrm{M}$, white columns to lowest dye concentration $5 \times 10^{-6} \mathrm{M}$. 
method, and CH film (CH new), prepared with the new method, about the efficiency in bleaching dye solution for each dye is reported. Traditional CH films have the property to swell in a large way when immersed in aqueous acidic solution compared with the same $\mathrm{CH}$ films both when it is immersed in basic (pH 12) aqueous solution or in $\mathrm{EtOH}$, and when it is neutralized by $\mathrm{NaOH}$ treatment [30]. In particular $\mathrm{CH}$ films treated with an acid dye solution swell in large way because of the electrostatic repulsion among $\mathrm{CH}$ protonated $\mathrm{NH}_{2}$ groups resulting in a reduced dyedecoloration rate which cannot be evaluated. Further it is known that an excess of acidity, considering a $\mathrm{pH}<2$, induces a strong competition of theanions, brought about by the dissociation of the acid used for $\mathrm{pH}$ control, and the sorption capacity significantly decreased [31]. It is also possible to observe the precipitation of almost all examined dyes due to their lower solubility at acidic pHs [20]. Consequently $\mathrm{CH}$ std and $\mathrm{CH}$ std film treated with an acid solution $(\mathrm{CH} \mathrm{pH} \mathrm{2)}$ appear useless for all considered dyes. On the contrary better results were obtained for traditional $\mathrm{CH}$ films treated with basic solutions $(\mathrm{CH} \mathrm{pH}$ 12), with EtOH $(\mathrm{CH} / \mathrm{ETOH})$ and neutralized with $\mathrm{NaOH}(\mathrm{CH} / \mathrm{NaOH})$ solution. This behavior could be attributed to the complete or partial neutralization of $\mathrm{NH}_{3}{ }^{+}$groups present on chitosan chains. Also the initial dye concentration is considered an important factor. The increase of the initial dye concentration determines an increase in the adsorption capacity of the adsorbent and this may be due to the high driving force for mass transfer at a high initial dye concentration [17]. A plateau region is established after some time and this plateau becomes much more evident for concentrated dye solution. In Figure 2(a) and Figure 2(b) are reported the decoloration rates referred to DY for $\mathrm{CH}$ std and $\mathrm{CH}$ new films respectively, after equilibrium has been reached. Surprisingly in all examined case spectroscopic data show the almost complete uptake of dye onto the different $\mathrm{CH}$ film. Better results are obtained for neutralized and long time dehydrated films. About the effect of initial dye concentration, in general the observed behavior appears to reflect the classical trend in which the amount of the dye adsorbed onto chitosan film increased with the increase of the initial concentration of dye solution under the same adsorbent amount. The only system for which it was not possible to verify this trend was the $\mathrm{CH}$ std after one day of dehydration (CH/ETOH 1d), since the film did not reach any equilibrium condition. About the time required for reaching the highest DY decoloration efficiency there is a wide range of possibility from 7 hrs (relative to the neutralized $\mathrm{CH}$ film at the lower initial dye concentration) until to 17 days in the case of new type of $\mathrm{CH}$ film without any treatment. In general the new CH films (Figure 2(b)) seem to need a lower contact time with the exception of the $\mathrm{CH}$ film used without modification which reaches the equilibrium in 17 days. The effect of dehydration treatment of $\mathrm{CH}$ films, realized by immersion of the film in EtOH for different times, results to be very interesting. In this case in 1 or 2 day occurs the complete removal of dye from both concentrated and diluted dye solutions, respect to the 4 or 5 days necessary for $\mathrm{CH}$ std in the same conditions. However, in spite of the long equilibrium time (17 days and 3 days for concentrated and dilute dye solution, respectively) needed for $\mathrm{CH}$ new in dye solution, those films show exceptional results due to the absence of collateral effects such as, for example, the acidification of the stained aqueous solution which always occurs using $\mathrm{CH}$ std films.

Of course, in term of equilibrium time and 100\% decoloration rate, treated $\mathrm{CH}$ new, in particular the film dehydrated 4 days $(\mathrm{CH} / \mathrm{ETOH} 4 \mathrm{~d})$, appears to be the best in its ability to remove DY dye from dilute and concentrated solutions. Furthermore, although the better adsorption characteristic are shown by $\mathrm{CH} / \mathrm{NaOH}$ films, the use of $\mathrm{CH}$ new films, dehydrated and not dehydrated with $\mathrm{EtOH}$, result more interesting because no further water washing cycles or neutralization are required and the long term absorption time ensure anyway an efficient decoloration of dyed solution.

In the Figure 2(c) and Figure 2(d) results related to DB dye are reported. Generally speaking, the $\mathrm{CH}$ film behavior appear very similar to the that shown in presence of DY, even though shorter equilibrium time is experimented in obtaining the same decoloration efficiency. In the case of $\mathrm{CH}$ std film the maximum of decoloration rate is reached for the neutralized $\mathrm{CH}$ std film $(\mathrm{CH} / \mathrm{NaOH})$, for long term dehydrate film $(\mathrm{CH} / \mathrm{ETOH} 4 \mathrm{~d})$ and for film in alkaline dye solution ( $\mathrm{CH} \mathrm{pH} \mathrm{12).} \mathrm{As} \mathrm{occurs} \mathrm{in} \mathrm{the} \mathrm{case} \mathrm{of} \mathrm{DY,} \mathrm{also} \mathrm{for} \mathrm{DB} \mathrm{the} \mathrm{decolorationrate} \mathrm{col-}$ lapses in acidic medium and, for long contact time, a dye precipitation has been also observed both for untreated $\mathrm{CH}$ std film and $\mathrm{CH}$ std film immersed in acid dye solution. The results obtained for $\mathrm{CH}$ std film previously neutralized in $\mathrm{NaOH}$ solution appear interesting. Only few hours are needed to completely remove the dye from solution both for concentrated and dilute solution. In the Figure 2(d) the effect of $\mathrm{CH}$ new film has been shown and not surprisingly the long time dehydrate film seems the best in term of decoloration rate. Unlike the system DY/CH new film, in this case the untreated $\mathrm{CH}$ new film appears the worst and a strong reduction of the decoloration rate has been observed. About the initial dye concentration effect it can observe that, reducing the dye concentration, the equilibrium time for an efficient removal of DB increments for all analyzed films. This could 
be attributed to a decrease in the driving force of the concentration gradient with the reduction of the initial dye concentration [14]. This effect is particularly evident for CH/ETOH $1 \mathrm{~d}$ and $4 \mathrm{~d}$ in which, decreasing the dye concentration, the time needed to efficiently remove the dye from aqueous solution increases very much passing from 2 days to 7 days for the $\mathrm{CH}$ film partially dehydrated and from $5 \mathrm{~h}$ to 3 days for the $\mathrm{CH}$ film completely dehydrate. However, as observed in the previous case, although the $\mathrm{CH} / \mathrm{NaOH}$ film seems to be the best in terms of contact time and efficiency in decoloration rate for both DB aqueous solutions, the use of CH/ETOH $4 \mathrm{~d}$ film results better avoiding the wash water cycle, which is time and water consuming, and the time needed to neutralize the film. In the panels e and $\mathbf{f}$ of the Figure 2 the results related to DR dye in presence of various $\mathrm{CH}$ films are reported. It is evident that the DR adsorption onto CH films is not so efficient for both examined film typologies (traditional and new method of film preparation) at high initial dye concentration. In particular only in the case of the standard $\mathrm{CH}$ film immersed in a $\mathrm{pH} 12$ dye aqueous solution, the system appears to be able to adsorb the dye with a relatively good efficiency at an initial DR concentration of $5 \times 10^{-5} \mathrm{M}$. The standard $\mathrm{CH}$ $\mathrm{pH} 12$ film can remove about the $60 \%$ of the dye, after 9 days. Conversely for diluted dye solutions good results have been obtained with an equilibrium time of $2-3$ days for both $\mathrm{CH}$ film typologies. Among the $\mathrm{CH}$ films prepared by means of the standard procedure, the $\mathrm{CH}$ film treated with $\mathrm{NaOH}$ seems to be the more functional system. While in the case of $\mathrm{CH}$ film prepared with the new method, the change in the film procedure preparation ensure the same efficiency after 2 or 3 days if $\mathrm{CH}$ new film was previously treated with $\mathrm{EtOH}$. As in the case of DB and DY, new CH/ETOH films show an excellent performance in removing DR dye with a decoloration rate of $100 \%$ provide that the dye concentration is maintained under $10^{-5} \mathrm{M}$.

As it is evident equilibrium time for reaching the highest decoloration rate is very different for the 3 studied dyes and it appears related to the dimensions and concentration of dyes, besides to the interactions between adsorbent film and dye molecules involving both electrostatic and Van der Waals interactions [26]. Although in general, the adsorption capacity increases with time, at certain point the system reaches a state of dynamic equilibrium between the amount of dye being adsorbed onto the material and the amount of dye desorbed from the adsorbent, then it is important to optimize this contact time, considering the efficiency of desorption and regeneration of the adsorbent. In the case of DY and DB, increasing initial dye concentration the result seems to get better although long contact times are required. Instead, decreasing the initial concentration it is observed a decrease of equilibrium time, together with a efficiency reduction around the $90 \%$ in almost all the DY systems, while better results have been achieved for DB. Only in the case of standard $\mathrm{CH} / \mathrm{NaOH}$ and $\mathrm{CHnew} / \mathrm{ETOH} 4 \mathrm{~d}$ the effect in term of decoloration efficiency appear to be unrelated with the dye concentration, for both dyes. This different behavior could be interpreted considering that, in general, during the adsorption process, the dye molecules progressively occupy the active sites present on the adsorbent surface, covering the film surface after some time and forming a monolayer dye coverage on the outer surface of the film. Obviously a large number of vacant surface sites are available for adsorption during the initial stage, and after a lapse of time, it is difficult to occupy there maining vacant surface sites due to repulsive forces between dye molecules adsorbed on the solid and those in the solution phase. Then after the dye adsorption from the solution to the adsorbent surface, the last stage of the adsorption process could be related to the diffusion of the dye-adsorbed molecules within the material. This last step can be strictly correlated to the sizes of the dye organic chains, to the amount and positioning of the sulfonate groups of the dyes and to the adsorption temperature [19] [31]-[33]. About this aspect, the adsorption from lower dye concentration appears to be more efficient for almost all analyzed dyes indicating that probably the rate determining step in reaching the equilibrium time is a combination between the rate in the active site occupation, which is directly correlated to the initial dye concentration [19], and the adsorbed dye molecules diffusion into the film, which is correlated to the dye molecular size and number of charged groups able to electrostatically interact with charged group on chitosan film [34].

Concerning kinetic treatments, as already said, the effect of initial dye concentration depends on the direct relation between the concentration of the dye and the available sites on the adsorbent surface for both typologies of film (CH std and new) and for all analyzed dye. In general the behavior of the Direct Yellow and Direct Blue is quite similar, while Direct Red differentiates. In the Figure 3 and Figure 4 (panel a and c) the adsorption profiles of the DY (Figure 3(a) and Figure 3(c)) and DB (Figure 4(a) and Figure 4(c)) in relation to the contact time are reported considering the two typologies of $\mathrm{CH}$ films. It is clear that the dye molecules progressively occupy the active sites of various $\mathrm{CH}$ films and, increasing the initial concentration of dye solution the adsorption capacity of $\mathrm{CH}$ films increases with time due to the driving force of the concentration gradient at least in the case of the higher dye concentration [19]. Furthermore it is also evident that equilibrium is reached in higher times for high 

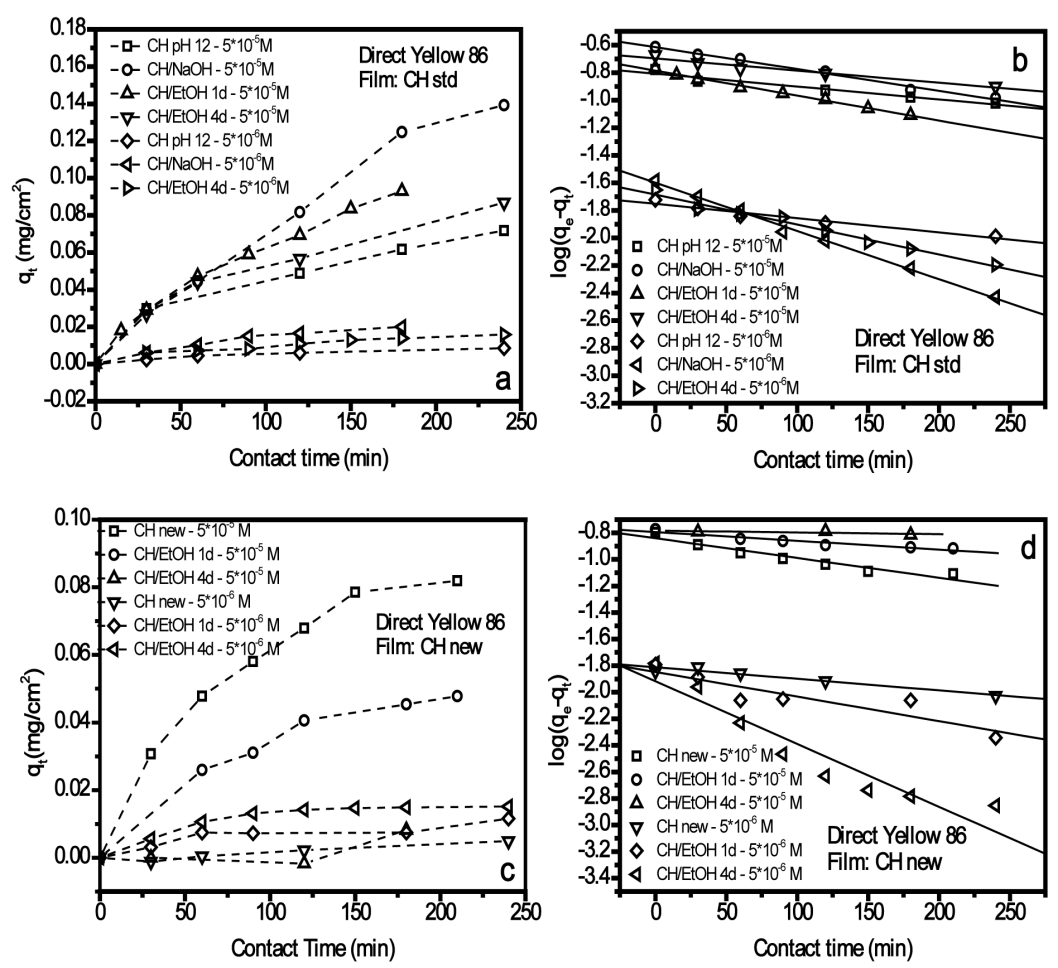

Figure 3. Amount of adsorption at time $t, q_{t}\left(\mathrm{mg} / \mathrm{cm}^{2}\right)$ versus time for DY in presence of CH std film (a) and CH new fil (c). Linearized first order plot for the absorption of DY in presence of CH std film (b) and CH new film (d).
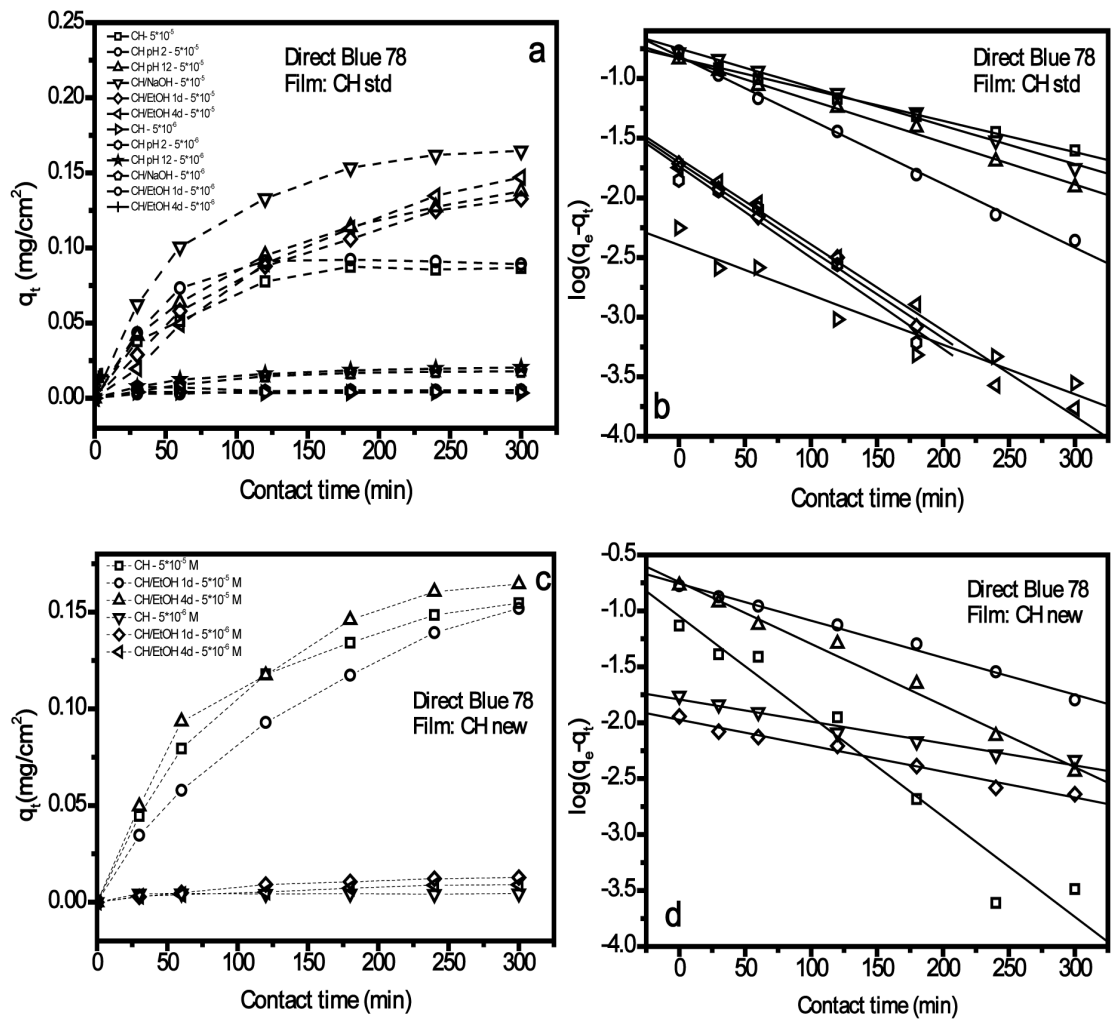

Figure 4. Amount of adsorption at time $t, q_{t}\left(\mathrm{mg} / \mathrm{cm}^{2}\right)$ versus time for DB in presence of CH std film (a) and CH new fil (c). Linearized first order plot for the absorption of DB in presence of $\mathrm{CH}$ std film (b) and CH new film (d). 
values of initial dye concentration. In the process of dye adsorption, initially the dye molecules have to be transferred at the adsorbent surface and then finally, they have to diffuse into the porous structure of the adsorbent [35]. Therefore, dye at higher initial concentrations will take relatively longer contact time to attain equilibrium due to higher amount of dye molecules respect to the number of available surface adsorption sites that in time decrease. Further another important parameter, which could affect the contact time for reaching the equilibrium, is the dye diffusion rate into the adsorbent pores. Thus the removal of dyes depends on the dye initial concentration and on the chemical characteristics of the dye [19] [31] [33] [34]. The amount of adsorbed dye at the equilibrium time reflects the maximum adsorption capacity of the adsorbent under chosen operating conditions. In particular, in the case of $\mathrm{CH}$ std film (Figure 3(a) and Figure 4(a)), the $q_{t}$ results to be very high for $\mathrm{CH} / \mathrm{NaOH}$ film and decreases diluting 10 times the solution for both dyes. About the $\mathrm{CH}$ new film the dye behavior differs more. In fact, while it is possible to individuate a marked difference in the trend of the $q_{t}$ at high and low initial dye concentration for DB (Figure 4(c)), in the case of DY the differences are less evident (Figure 3(c)). The untreated $\mathrm{CH}$ new film $(\mathrm{CH})$ presents the highest dye uptake during the adsorption first phase for the DY (Figure 3(c)). For DB (Figure 4(c)), the CH/ETOH film shows the higher $q_{t}$. This could indicate that, after an initial relatively rapid dye adsorption under the dye concentration gradient (initial dye concentration effect), the saturation of the $\mathrm{CH}$ film vacant sites takes place slowing down the further dye adsorption in function of different chemical structure of the two dyes. The rapid initial phase is different for the two analyzed dyes and it may last from several minutes to few hours, while the following slow stage may continue for hours or days reaching a plateau. The behavior of system $\mathrm{CH}$ film/dye appears very interesting at low dye concentration where the plateau is absent. This can be interpreted considering that at low dye concentration the ratio of initial number of dye molecules to the active sites on the adsorbent film is low and subsequently the fractional adsorption becomes independent from initial dye concentration.

Different results have been obtained in the case of DR. In Figure 5 (panels a and c) the adsorption profiles
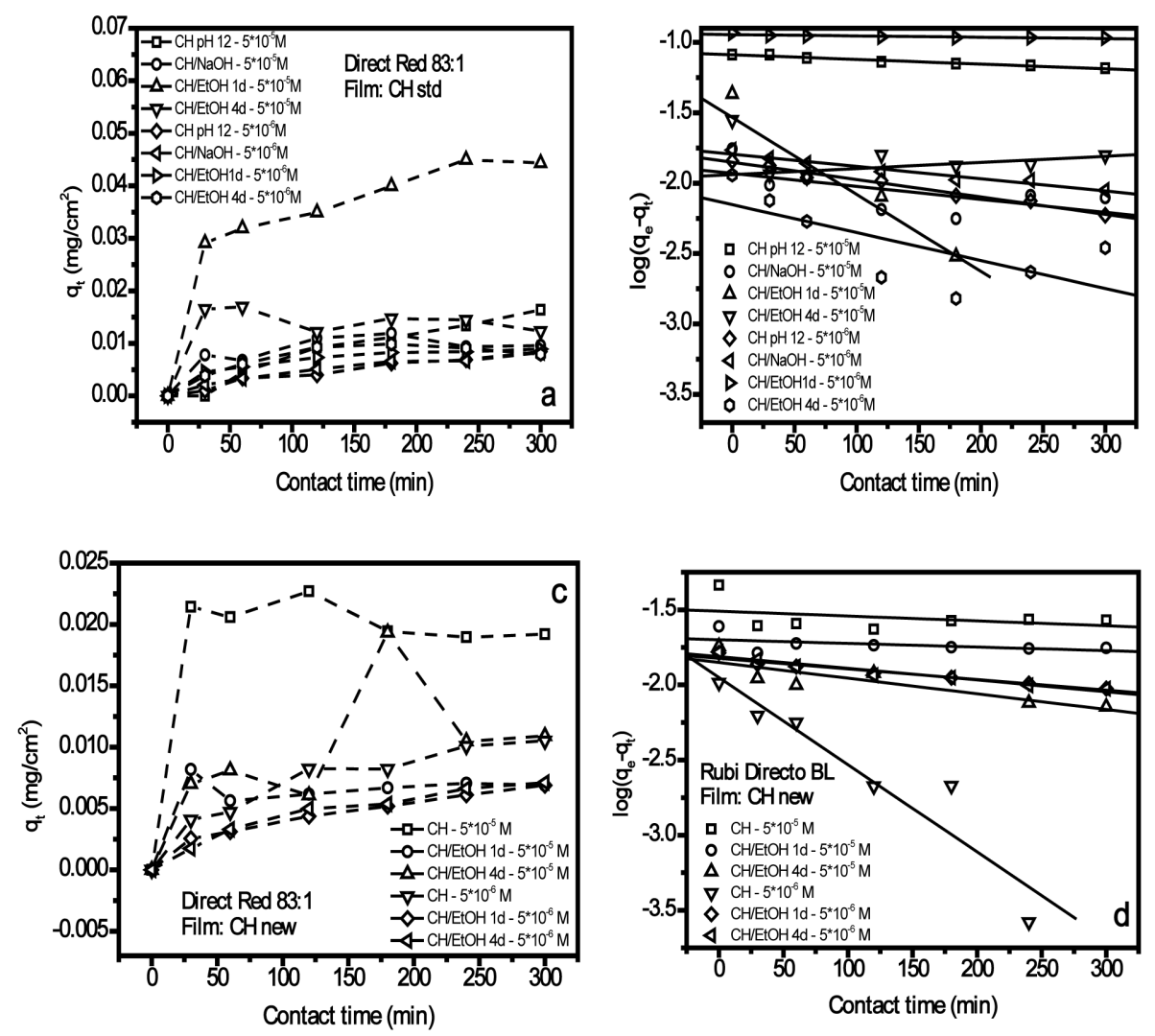

Figure 5. Amount of adsorption at time $t, q_{t}\left(\mathrm{mg} / \mathrm{cm}^{2}\right)$ versus time for DR in presence of CH std film (a) and CH new fil (c). Linearized first order plot for the absorption of DR in presence of CH std film (b) and CH new film (d). 
of the red dye in relation to the contact time for various $\mathrm{CH}$ films are reported. It is evident that there are no marked variation of the $\mathrm{q}_{\mathrm{t}}$ with the initial dye concentration for both examined film typologies, as already observed for the decoloration rate (panel $\mathbf{e}$ and $\mathbf{f}$ in Figure 2). For the $\mathrm{CH}$ std film type (Figure 5(a)), the $\mathrm{CH}$ film treated with ETOH for 1 day (CH/ETOH 1d) shows the highest dye uptake for a dye concentration of $5 \times 10^{-5}$ $\mathrm{M}$. While, for the same dye concentration, $\mathrm{CH}$ film without further treatment ( $\mathrm{CH}$ in Figure 5(c)) results to have the highest adsorption capacity within the $\mathrm{CH}$ films prepared with the new method. No significant differences have been observed for the other film types in function of the initial dye concentration indicating that the effect of mass transfer could be considered irrelevant for the system DR/CH film. In order to investigate the mechanism of adsorption, a pseudo-first order and a pseudo-second order adsorption model has been used to test dynamical experimental data. The pseudo-first order and pseudo-second order kinetic models assume that the adsorption is a pseudo-chemical reaction. Many kinetic models have been developed with the aim to find intrinsic kinetic adsorption parameters [19]. Traditionally, data on kinetics of adsorption are described following the expressions originally given by Lagergren, which has adapted the general Langmuir rate equation to special cases [36] [37]. In Figures 3-5 (panels $\mathbf{b}$, for $\mathrm{CH}$ std and $\mathbf{d}$, for $\mathrm{CH}$ new) the trends of adsorption process calculated by means of the Lagergren model are reported for the different $\mathrm{CH}$ film typologies. An ideal adsorbent for waste water pollution remediation must not only present a large adsorbate capacity but also a fast adsorption rate. Therefore, the adsorption rate is another important parameter for selecting the adsorbent material and adsorption kinetics have to be considered since they explain how fast the chemical reaction occurs and also provides information on the factors affecting the reaction rate. About the kinetics of adsorption, differences in chitosan type, preparation, dyes and methodology examined makes any comparison of results difficult [19]. Table 1, Table 2 and Table 3 show the

Table 2. Comparison of the pseudo-first-order, pseudo-second-order adsorption rate constants and calculated and experimental $q_{e}$ values obtained at different Direct Blue 78 concentrations.

\begin{tabular}{|c|c|c|c|c|c|c|c|c|}
\hline \multirow{2}{*}{ Film } & \multirow{2}{*}{$\begin{array}{c}q_{e, \text { th }} \\
\left(\mathrm{mg} / \mathrm{cm}^{2}\right)\end{array}$} & \multirow{2}{*}{$\begin{array}{c}q_{e, \exp } \\
\left(\mathrm{mg} / \mathrm{cm}^{2}\right)\end{array}$} & \multicolumn{3}{|c|}{ Pseudo-first order } & \multicolumn{3}{|c|}{ Pseudo-second order } \\
\hline & & & $\begin{array}{c}q_{e, \text { calc }} \\
\left(\mathrm{mg} / \mathrm{cm}^{2}\right)\end{array}$ & $\mathrm{K}\left(\min ^{-1}\right)$ & $\mathrm{R}^{2}$ & $\begin{array}{c}q_{e, \text { calc }} \\
\left(\mathrm{mg} / \mathrm{cm}^{2}\right)\end{array}$ & $\mathrm{K}$ & $\mathrm{R}^{2}$ \\
\hline \multicolumn{9}{|c|}{ DB/CH new } \\
\hline \multicolumn{9}{|c|}{ Concentrated dye } \\
\hline $\mathrm{CH}$ & 0.16 & 0.074 & 0.089 & $(2.00 \pm 0.1) \times 10^{-2}$ & 0.97 & 0.21 & $(4.66 \pm 1.08) \times 10^{-2}$ & 0.99 \\
\hline $\mathrm{CH} / \mathrm{EtOH} 1 \mathrm{~d}$ & 0.17 & 0.17 & 0.17 & $(8.00 \pm 0.1) \times 10^{-3}$ & 0.99 & 0.25 & $(2.04 \pm 0.35) \times 10^{-2}$ & 0.99 \\
\hline $\mathrm{CH} / \mathrm{EtOH} 4 \mathrm{~d}$ & 0.17 & 0.17 & 0.18 & $(1.50 \pm 0.03) \times 10^{-2}$ & 0.99 & 0.22 & $(4.81 \pm 1.42) \times 10^{-2}$ & 0.99 \\
\hline \multicolumn{9}{|c|}{ Diluted dye } \\
\hline CH/EtOH 1d & 0.017 & 0.017 & 0.016 & $(4.50 \pm 0.2) \times 10^{-3}$ & 0.99 & 0.021 & $(25.75 \pm 7.23) \times 10^{-2}$ & 0.99 \\
\hline $\mathrm{CH} / \mathrm{EtOH} 4 \mathrm{~d}$ & 0.017 & 0.011 & 0.11 & $(5.00 \pm 0.02) \times 10^{-3}$ & 0.99 & 0.013 & $(58.36 \pm 25.32) \times 10^{-2}$ & 0.96 \\
\hline \multicolumn{9}{|c|}{$\mathrm{DB} / \mathrm{CH}$ std } \\
\hline \multicolumn{9}{|c|}{ Concentrated Dye } \\
\hline $\mathrm{CH}$ pH 12 & 0.18 & 0.16 & 0.15 & $(60.00 \pm 0.09) \times 10^{-3}$ & 0.99 & 0.19 & $(46.03 \pm 8.96) \times 10^{-3}$ & 1 \\
\hline $\mathrm{CH} / \mathrm{NaOH}$ & 0.17 & 0.17 & 0.15 & $(12.00 \pm 0.02) \times 10^{-2}$ & 0.99 & 0.20 & $(81.15 \pm 20.11) \times 10^{-3}$ & 1 \\
\hline $\begin{array}{c}\mathrm{CH} / \mathrm{EtOH} \\
1 \mathrm{~d}\end{array}$ & 0.17 & 0.14 & 0.15 & $(8.00 \pm 0.10) \times 10^{-3}$ & 0.99 & 0.21 & $(27.56 \pm 6.42) \times 10^{-3}$ & 0.99 \\
\hline $\begin{array}{c}\mathrm{CH} / \mathrm{EtOH} \\
4 \mathrm{~d}\end{array}$ & 0.17 & 0.16 & 0.18 & $(8.00 \pm 0.1) \times 10^{-3}$ & 0.99 & 0.41 & $(4.90 \pm 1.64) \times 10^{-3}$ & 0.85 \\
\hline \multicolumn{9}{|c|}{ Diluted dye } \\
\hline $\mathrm{CH}$ pH 12 & 0.018 & 0.019 & 0.019 & $(2.00 \pm 0.04) \times 10^{-2}$ & 0.99 & 0.025 & $(61.84 \pm 13.94) \times 10^{-2}$ & 1 \\
\hline $\mathrm{CH} / \mathrm{NaOH}$ & 0.017 & 0.018 & 0.021 & $(2.00 \pm 0.04) \times 10^{-2}$ & 0.99 & 0.026 & $(34.60 \pm 13.96) \times 10^{-2}$ & 0.98 \\
\hline $\mathrm{CH} / \mathrm{EtOH} 1 \mathrm{~d}$ & 0.017 & 0.0056 & 0.004 & $(9.00 \pm 0.50) \times 10^{-3}$ & 0.97 & 0.006 & $4.01 \pm 1.93$ & 0.99 \\
\hline $\begin{array}{c}\mathrm{CH} / \mathrm{EtOH} \\
4 \mathrm{~d}\end{array}$ & 0.017 & 0.014 & 0.018 & $(2.00 \pm 0.10) \times 10^{-2}$ & 0.98 & 0.03 & $7.69 \pm 2.96$ & 0.92 \\
\hline
\end{tabular}


Table 3. Comparison of the pseudo-first-order, pseudo-second-order adsorption rate constants and calculated and experimental $q_{e}$ values obtained at different Direct Red 83:1 concentrations.

\begin{tabular}{|c|c|c|c|c|c|c|c|c|}
\hline \multirow{2}{*}{ Film } & \multirow{2}{*}{$\begin{array}{c}q_{e, \text { th }} \\
\left(\mathrm{mg} / \mathrm{cm}^{2}\right)\end{array}$} & \multirow{2}{*}{$\begin{array}{c}q_{e, \exp } \\
\left(\mathrm{mg} / \mathrm{cm}^{2}\right)\end{array}$} & \multicolumn{3}{|c|}{ Pseudo-first order } & \multicolumn{3}{|c|}{ Pseudo-second order } \\
\hline & & & $\begin{array}{c}q_{e, \text { calc }} \\
\left(\mathrm{mg} / \mathrm{cm}^{2}\right)\end{array}$ & $\mathrm{K}\left(\min ^{-1}\right)$ & $\mathrm{R}^{2}$ & $\begin{array}{c}q_{e, \text { calc }} \\
\left(\mathrm{mg} / \mathrm{cm}^{2}\right)\end{array}$ & K & $\mathrm{R}^{2}$ \\
\hline \multicolumn{9}{|c|}{ DR/CH new } \\
\hline & & & & Concentrated dye & & & & \\
\hline $\mathrm{CH}$ & 0.15 & 0.046 & 0.030 & $(7.46 \pm 3.65) \times 10^{-4}$ & 0.36 & / & / & I \\
\hline $\mathrm{CH} / \mathrm{EtOH} 1 \mathrm{~d}$ & 0.16 & 0.025 & 0.019 & $(5.58 \pm 1.99) \times 10^{-4}$ & 0.48 & 0.007 & $17.36 \pm 16.29$ & 0.99 \\
\hline $\mathrm{CH} / \mathrm{EtOH} 4 \mathrm{~d}$ & 0.16 & 0.018 & 0.014 & $(23.95 \pm 3.13) \times 10^{-4}$ & 0.85 & I & / & I \\
\hline \multicolumn{9}{|c|}{ Diluted dye } \\
\hline $\mathrm{CH}$ & 0.016 & 0.010 & 0.011 & $(133 \pm 10) \times 10^{-4}$ & 0.94 & 0.013 & $0.90 \pm 0.39$ & 0.98 \\
\hline $\mathrm{CH} / \mathrm{EtOH} 1 \mathrm{~d}$ & 0.016 & 0.016 & 0.015 & $(16.31 \pm 0.74) \times 10^{-4}$ & 0.97 & 0.009 & $1.06 \pm 0.45$ & 0.98 \\
\hline $\mathrm{CH} / \mathrm{EtOH} 4 \mathrm{~d}$ & 0.017 & 0.016 & 0.015 & $(17.91 \pm 0.85) \times 10^{-4}$ & 0.97 & 0.010 & $0.22 \pm 0.74$ & 0.99 \\
\hline \multicolumn{9}{|c|}{$\mathrm{DR} / \mathrm{CH}$ std } \\
\hline \multicolumn{9}{|c|}{ Concentrated Dye } \\
\hline CH pH 12 & 0.15 & 0.081 & 0.081 & $(7.66 \pm 0.28) \times 10^{-4}$ & 0.98 & 0.04 & $(55.65 \pm 51.85) \times 10^{-2}$ & 0.95 \\
\hline $\mathrm{CH} / \mathrm{NaOH}$ & 0.15 & 0.017 & 0.012 & $(21.22 \pm 4.99) \times 10^{-4}$ & 0.64 & 0.01 & $13.98 \pm 22.21$ & 0.98 \\
\hline $\begin{array}{c}\mathrm{CH} / \mathrm{EtOH} \\
1 \mathrm{~d}\end{array}$ & 0.15 & 0.043 & 0.029 & $(1.26 \pm 0.11) \times 10^{-2}$ & 0.94 & 0.05 & $(61.09 \pm 33.03) \times 10^{-2}$ & 0.99 \\
\hline $\begin{array}{c}\mathrm{CH} / \mathrm{EtOH} \\
4 \mathrm{~d}\end{array}$ & 0.16 & 0.028 & 0.011 & $(10.02 \pm 2.23) \times 10^{-4}$ & 0.70 & 0.013 & I & 0.99 \\
\hline \multicolumn{9}{|c|}{ Diluted dye } \\
\hline CH pH 12 & 0.016 & 0.014 & 0.014 & $(28.32 \pm 0.81) \times 10^{-4}$ & 0.98 & 0.023 & $(8.27 \pm 3.46) \times 10^{-2}$ & 0.70 \\
\hline $\mathrm{CH} / \mathrm{NaOH}$ & 0.016 & 0.017 & 0.016 & $(20.12 \pm 0.82) \times 10^{-4}$ & 0.98 & 0.012 & $(54.86 \pm 18.57) \times 10^{-2}$ & 0.98 \\
\hline CH/EtOH 1d & 0.016 & 0.012 & 0.113 & $(21.72 \pm 2.44) \times 10^{-5}$ & 0.86 & 0.01 & $2.31 \pm 0.75$ & 0.99 \\
\hline $\begin{array}{c}\mathrm{CH} / \mathrm{EtOH} \\
4 \mathrm{~d}\end{array}$ & 0.016 & 0.011 & 0.007 & $(45.60 \pm 9.04) \times 10^{-4}$ & 0.70 & 0.009 & $5.24 \pm 5.25$ & 0.97 \\
\hline
\end{tabular}

values of the pseudo-first order and pseudo-second order models together with the coefficient of correlation $\left(R^{2}\right)$ and the average relative error for the three analyzed dyes in relation to the different types of $\mathrm{CH}$ films. Assuming a pseudo-first order kinetics, the calculated $q_{e}\left(q_{e, \text { calc }}\right)$ values are agree with the experimental $q_{e}$ values. This suggests that the adsorption of dye follows a first-order kinetics for all analyzed $\mathrm{CH}$ films. Good correlation coefficients have been also obtained for almost all examined dyes. In the case in which dye have been completely absorbed, the great efficiency shows by the used $\mathrm{CH}$ films is demonstrated by the comparison between the experimental $q_{e, \text { exp }}$ and the theoretical values of expected $q_{e, t h}$ calculated applying the following equation:

$$
q_{e, t h}=\frac{C_{0}}{A_{\mathrm{CH}}} \times V
$$

where $q_{e, t h}\left(\mathrm{mg} / \mathrm{cm}^{2}\right)$ is the amount of adsorbed dye at the equilibrium, $C_{0}$ is the initial dye concentration spectro photo metrically evaluated, $A_{\mathrm{CH}}$ is the geometrical area of the used $\mathrm{CH}$ film in $\mathrm{cm}^{2}$ and $V(\mathrm{~L})$ is the dye solution volume. This equation derives from the application of Equation (2) to the calculation of the $q_{e}$ values hypothesizing that all the dye has been adsorbed onto the $\mathrm{CH}$ film, whereby the amount of dye present in solution at the equilibrium is null $\left(C_{t}=C_{e} \approx 0 \mathrm{M}\right)$. More the calculated $q_{e, t h}$ is closed to $q_{e, \text { exp }}$, the greater is the efficiency of various typologies of analyzed CH film. Comparing the obtained $q_{e, t h}$ values for DY and DB with the $q_{e, e x p}$ (shown in Table 1 and Table 2), an excellent dye uptake for each employed dye concentration relative to different film treatment is suggested. 
However, as already observed by the analysis of the decoloration rate (Figure 2), not all CH film treatments improve the adsorption efficiency. For example there is a very high difference between the $q_{e, t h}$ and the $q_{e, \text { exp }}$ in the case of the untreated $\mathrm{CH}$ film prepared with the modified procedure respect to the adsorption of $\mathrm{DB}$, indicating that this new typology of $\mathrm{CH}$ film, if it is not dehydrated by ethanolic treatment, presents a low affinity for that dye. The obtained kinetic constant values suggest the effect of mass transfer, the initial dye concentration and affinity of DB respect to DY towards CH films. Figure 5(b) and Figure 5(c) show the linear form of the pseudo-first to the DR/CH film system. Only for diluted dye, the obtained $q_{e}$ (Table 3 ) suggest an excellent dye uptake and kinetic constant values (Table 3 ) result to be in agreement with the $q_{t}$ values. Calculated $q_{e, \text { calc }}$ values are agree with the experimental $q_{e, e x p}$ values, also in this case.

Concerning the obtained results, probably an explication of this behavior could be found considering the concept of the point of zero charge $\left(\mathrm{pH}_{\mathrm{pzc}}\right)$, indicative of the surface adsorption ability and of the type of surface active centers, applied to $\mathrm{CH}$ film. In particular the point of zero charge (pzc) is the $\mathrm{pH}$ value at which the surfacecharge is zero and it is typically used to quantify or define the electrokinetic properties of a surface. Consequently due to the presence of functional groups on the surface of the absorbent material, cationic or anionic dye will be absorbed at $\mathrm{pH}>\mathrm{pH}_{\mathrm{pzc}}$ or $\mathrm{pH}<\mathrm{pH}_{\mathrm{pzc}}$ [19]

The reported $\mathrm{pH}_{\mathrm{pzc}}$ value for chitosan is 6.6 [38] so that the adsorption of a cationic dye may be favored at $\mathrm{pH}>$ $\mathrm{pH}_{\mathrm{pzc}}$, whereas anionic dye adsorption would be favored at $\mathrm{pH}<\mathrm{pH}_{\mathrm{pzc}}$ where the $\mathrm{CH}$ surface becomes positively charged [18]. In our case the maximum dye uptake has been observed for all examined textile dyes when $\mathrm{CH}$ films have been previously dehydrated with $\mathrm{EtOH}$, when treated in alkaline medium and when neutralized. However the amino groups of chitosan are protonated under acidic conditions, whereas in alkaline medium and/or in ethanolic solution, they result mainly unprotonated or partially protonated [26]. All employed dye are negatively charged and in an alkaline medium their charge density increases, further the parallel effect of EtOH is the increase of the hydrophobic character of $\mathrm{CH}$ films. This suggests that, in these dye/CH film systems, the electrostatic interactions are very weak and the adsorption processes are controlled mainly by physical forces such as Van der Waals forces, hydrogen bonds, polarity, dipole-dipole bonds, $\pi-\pi$ interaction, etc [18]. In fact the ethanolic treatment of $\mathrm{CH}$ film induces a reorganization of $\mathrm{CH}$ chains within the film in a more favorable thermodynamic conformation since $\mathrm{CH}$ is insoluble in EtOH. In particular ethanol treated $\mathrm{CH}$ films result partially protonated, containing anhydrous "annealed” polymorph of chitosan and "type II" crystals of chitosan acetate, due to the simultaneous, but incomplete removal of the residual acid and water molecules from the films by immersion in ethanol [26]. The CH chain reorganization induced by EtOH favors the formation of hydrophobic domainsin chitosan [39]. This behavior leads to think that dye adsorption process would be favored in acidic medium where $\mathrm{CH}$ is completely protonated, but probably the excessive swelling of $\mathrm{CH}$ films in this case determine too weak $\mathrm{CH}$ film/dye interactions. It was not excluded the role of the lower solubility of dye in this condition. The worst results have been obtained in the case of DR, where the presence of $\mathrm{OH}$ moieties in the dye structure obstacle hydrophobic interactions between dye and $\mathrm{CH}$ films, especially if dye is present at high concentration. The CH film saturation in this case is achieved faster than for DY and DB. The effect of EtOH ensures the presence of hydrophobic interaction between anthracene and benzene moieties, and polar effect $\mathrm{OH}$ groups working mainly in opposition. More detailed analysis about the interactions CH/dyes by means of FTIRATR, morphology and mechanism of desorption and recycle are in progress. Also elucidation of EtOH and $\mathrm{NaOH}$ effects will be considered.

\section{Conclusion}

In this study, the adsorption of three anionic dyes, Direct Red 83:1, Direct Yellow 86 and Direct Blue 78, onto different types of $\mathrm{CH}$ films has been investigated focusing on equilibrium and kinetic process. The adsorption experiments confirm that $\mathrm{CH}$ modified films are effective for the adsorption of basic dye from aqueous solution. The kinetic studies show that the adsorption onto the different typologies $\mathrm{CH}$ follows a first order kinetics and Langergren model has been successfully applied. The adsorption of dyes onto $\mathrm{CH}$ film results to be dependent on various parameters such as aqueous solution $\mathrm{pH}$, contact time and initial dye concentration. In particular, for $\mathrm{CH}$ std type films, good results have been obtained when the film is neutralized with $\mathrm{NaOH}$ (it is immersed in alkaline dye solution), and when it is treated previously with an ethanolic solution for 4 days. However changing the method of $\mathrm{CH}$ film preparation there is a remarkable improvement, especially when the film is treated with $\mathrm{EtOH}$. The new method developed for preparing neutral $\mathrm{CH}$ films allows avoiding many washing cycles reduc- 
ing the total cost of the process. Regarding the dye/CH film interaction DY and DB appear much more akin to $\mathrm{CH}$ films than DR, probably due to their ability in establishing hydrophobic interactions, which are prevented in the case of DR due to the presence of various OH groups.

\section{Acknowledgements}

This study was supported by the European "DYES4EVER" (Demonstration of cyclodextrin techniques in treatment of waste water in textile industry to recover and reuse textile dyes, LIFE12 ENV/ES/000309) within the LIFE+2012 program "Environment Policy and Governance project application". We gratefully acknowledge the skilful and excellent technical assistance of Mr. Sergio Nuzzo.

\section{References}

[1] Savenije, H.H.G. (2002) Why Water Is Not an Ordinary Economic Good, or Why the Girl Is Special. Physics and Chemistry of the Earth, 27, 741-744. http://dx.doi.org/10.1016/S1474-7065(02)00060-8

[2] Van der Oost, R., Beyer, J. and Vermeulen, N.P.E. (2003) Fish Bioaccumulation and Biomarkers in Environmental Risk Assessment: A Review. Environmental Toxicology and Pharmacology, 13, 57-149. http://dx.doi.org/10.1016/S1382-6689(02)00126-6

[3] Robinson, T., McMullan, G., Marchant, R. and Nigam, P. (2001) Remediation of Dyes in Textile Effluent: A Critical Review on Current Treatment Technologies with a Proposed Alternative. Bioresource Technology, 77, 247-255. http://dx.doi.org/10.1016/S0960-8524(00)00080-8

[4] Mirmohseni, A., SeyedDorraji, M.S., Figoli, A. and Tasselli, F. (2012) Chitosan Hollow Fibers as Effective Biosorbent toward Dye: Preparation and Modeling. Bioresource Technology, 121, 212-220. http://dx.doi.org/10.1016/j.biortech.2012.06.067

[5] Crini,G. (2005) Recent Developments in Polysaccharide-Based Materials Used as Adsorbents in Wastewater Treatment. Progress in Polymer Science, 30, 38-70. http://dx.doi.org/10.1016/j.progpolymsci.2004.11.002

[6] Goncharuk,V.V., Kucheruk, D.D., Kochkodan, V.M., and Badekha, V.P. (2002) Removal of Organic Substances from Aqueous Solutions by Reagent Enhanced Reverse Osmosis. Desalination, 143, 45-51. http://dx.doi.org/10.1016/S0011-9164(02)00220-5

[7] Lopez, F.A., Martin, M.I., Pèrez, C., Lopez-Delgado, A. and Alguacil, F.J. (2003) Removal of Copper Ions from Aqueous Solutions by a Steel-Making By-Product. Water Research, 37, 3883-3890. http://dx.doi.org/10.1016/S0043-1354(03)00287-2

[8] Aksu, Z. (2005) Application of Biosorption for the Removal of Organic Pollutants: A Review. Process Biochemistry, 40, 997-1026. http://dx.doi.org/10.1016/j.procbio.2004.04.008

[9] Park, D., Yun, Y. and Park, J.M. (2012) The Past, Present, and Future Trends of Biosorption. Biotechnology and Bioprocess Engineering, 15, 86-102. http://dx.doi.org/10.1007/s12257-009-0199-4

[10] Reddy, D.H.K. and Lee, S.-M. (2013) Application of Magnetic Chitosan Composites for the Removal of Toxic Metal and Dyes from Aqueous Solutions. Advances in Colloid and Interface Science, 201-202, 68-93.

[11] Rabea, E.I., Badawy, M.E.T., Stevens, C.V., Smagghe, G. and Steurbaut, W. (2003) Chitosan as Antimicrobial Agent: Applications and Mode of Action. Biomacromolecules, 4, 1457-1465. http://dx.doi.org/10.1021/bm034130m

[12] Moczek, L. and Nowakowska, M. (2007) Novel Water-Soluble Photosensitizers from Chitosan. Biomacromolecules, 8, 433-438. http://dx.doi.org/10.1021/bm060454+

[13] Haldorai, Y. and Shim, J.J. (2014) An Efficient Removal of Methyl Orange Dye from Aqueous Solution by Adsorption onto Chitosan/MgO Composite: A Novel Reusable Adsorbent. Applied Surface Science, 292, 447-453. http://dx.doi.org/10.1016/j.apsusc.2013.11.158

[14] Crini, G. and Badot, P.M. (2008) Application of Chitosan, a Natural Aminopolysaccharide, for Dye Removal from Aqueous Solutions by Adsorption Processes Using Batch Studies: A Review of Recent Literature. Progress in Polymer Science, 33, 399-447. http://dx.doi.org/10.1016/j.progpolymsci.2007.11.001

[15] Jiang, X., Sun, Y., Liu, L., Wang, S. and Tian, X. (2014) Adsorption of C.I. Reactive Blue 19 from Aqueous Solutions by Porous Particles of the Grafted Chitosan. Chemical Engineering Journal, 235, 151-157. http://dx.doi.org/10.1016/j.cej.2013.09.001

[16] Nandi, B., Goswami, A. and Purkait, M. (2009) Removal of Cationic Dyes from Aqueous Solutions by Kaolin: Kinetic and Equilibrium Studies. Applied Clay Science, 42, 583-590. http://dx.doi.org/10.1016/j.clay.2008.03.015

[17] Bulut, Y. and Aydın, H. (2006) A Kinetics and Thermodynamics Study of Methylene Blue Adsorption on Wheat Shells. Desalination, 194, 259-267. http://dx.doi.org/10.1016/j.desal.2005.10.032 
[18] Yagub, M.T., Tushar Kanti, S., Afroze, S. and Ang, H.M. (2014) Dye and Its Removal from Aqueous Solution by Adsorption: A Review. Advances in Colloid and Interface Science, 209, 172-184. http://dx.doi.org/10.1016/j.cis.2014.04.002

[19] Gupta, V. (2009) Application of Low-Cost Adsorbents for Dye Removal—A Review. Journal of Environmental Management, 90, 2313-2342. http://dx.doi.org/10.1016/j.jenvman.2008.11.017

[20] Clarke, E. and Anliker, R. (1980) Organic Dyes and Pigments. The Handbook of Environmental Chemistry, 3, $181-215$.

[21] Pinheiro, H.M., Touraud, E. and Thomas, O. (2004) Aromatic Amines from Azo Dye Reduction: Status Review with Emphasis on Direct UV Spectrophotometric Detection in Textile Industry Wastewaters. Dyes and Pigments, 61, 121139. http://dx.doi.org/10.1016/j.dyepig.2003.10.009

[22] Robinson, T., McMullan, G., Marchant, R. and Nigam, P. (2001) Remediation of Dyes in Textile Effluent: A Critical Review on Current Treatment Technologies with a Proposed Alternative. Bioresource Technology, 77, 247-255. http://dx.doi.org/10.1016/S0960-8524(00)00080-8

[23] Verma, Y. (2001) Acute Toxicity Assessment of Textile Dyes and Textile and Dye Industrial Effluents Using Daphnia Magna Bioassay. Toxicology and Industrial Health, 24, 491-500. http://dx.doi.org/10.1177/0748233708095769

[24] Krajewska, B., Leszko, M. and Zaborska, W. (1990) Urease Immobilized on Chitosan Membrane: Preparation and Properties. Journal of Chemical Technology and Biotechnology, 48, 337-350. http://dx.doi.org/10.1002/jctb.280480309

[25] Krajewska, B. (1991) Chitin and Its Derivatives as Supports for the Immobilization of Enzymes. Acta Biotechnologica, 11, 269-277. http://dx.doi.org/10.1002/abio.370110319

[26] Fang, R., Cheng, X.S., Fu, J. and Zheng, Z.B. (2009) Research on the Graft Copolymerization of EH-Lignin with Acrylamide. Natural Science, 1, 17-22. http://dx.doi.org/10.4236/ns.2009.11004

[27] Khataee, A.R., Pons, M.S. and Zahra, O. (2009) Photocatalytic Degradation of Three Azo Dyes Using Immobilized $\mathrm{TiO}_{2}$ Nanoparticles on Glass Plates Activated by UV Light Irradiation: Influence of Dye Molecular Structure. Journal of Hazardous Materials, 168, 451-457. http://dx.doi.org/10.1016/j.jhazmat.2009.02.052

[28] Jin, J., Li, L.S., Zhang, Y.J., Tian, Y.Q., Jiang, S., Zhao, Y., Bai, Y. and Li, T.J. (1998) Characterization and Structure of Side-On Azo Copolymers in Langmuir-Blodgett Films. Langmuir, 14, 5231-5236. http://dx.doi.org/10.1021/la980056v

[29] Karukstis, K.K., Perelman, L.A. and Wong, W.K. (2002) Spectroscopic Characterization of Azo Dye Aggregation on Dendrimer Surfaces. Langmuir, 18, 10363-10371. http://dx.doi.org/10.1021/la020558f

[30] He, Q., Ao, Q., Gong, Y.D. and Zhang, X.F. (2011) Preparation of Chitosan Films Using Different Neutralizing Solutions to Improve Cell Compatibility. Journal of Materials Science: Materials in Medicine, 22, 2791-2802. http://dx.doi.org/10.1007/s10856-011-4444-y

[31] Gibbs, G., Tobin, J.M. and Guibal, E. (2003) Sorption of Acid Green 25 on Chitosan: Influence of Experimental Parameters on Uptake Kinetics and Sorption Isotherms. Journal of Applied Polymer Science, 90, 1073-1080. http://dx.doi.org/10.1002/app.12761

[32] Cestari, A.R., Vieira, E.F.S., Pinto, A.A. and Lopes, E.C.N. (2005) Multistep Adsorption of Anionic Dyes on Silica/Chitosan Hybrid: 1. Comparative Kinetic Data from Liquid- and Solid-Phase Models. Journal of Colloid and Interface Science, 292, 363-372. http://dx.doi.org/10.1016/j.jcis.2005.05.096

[33] Cestari, A.R., Vieira, E.F.S., Dos Santos, A.G.P., Mota, J.A. and De Almeida, V.P. (2004) Adsorption of Anionic Dyes on Chitosan Beads. 1. The Influence of the Chemical Structures of Dyes and Temperature on the Adsorption Kinetics. Journal of Colloid and Interface Science, 280, 380-386. http://dx.doi.org/10.1016/j.jcis.2004.08.007.

[34] Maghami, G.G. and Roberts, G.A. (1988) Studies on the Interaction of Anionic Dyes on Chitosan. Macromolecular Chemistry and Physics, 189, 239-243.

[35] Shouman, M.A., Khedr, S.A. and Attia, A.A. (2012) Basic Dye Adsorption on Low Cost Biopolymer: Kinetic and Equilibrium Studies. IOSR Journal of Applied Chemistry, 2, 27-36.

[36] Putnis, A. (1995) Introduction to Mineral Sciences. Cambridge University Press, Melbourne.

[37] Chiou, M.S., Ho, P.Y. and Li, H.Y. (2004) Adsorption of Anionic Dyes in Acid Solutions Using Chemically Cross-Linked Chitosan Beads. Dyes and Pigments, 60, 69-84. http://dx.doi.org/10.1016/S0143-7208(03)00140-2

[38] Ali, H. (2010) Biodegradation of Synthetic Dyes-A Review. Water, Air, \& Soil Pollution, 213, 251-273. http://dx.doi.org/10.1007/s11270-010-0382-4

[39] Philippova, O.E., Volkov, E.G., Sitnikova, N.L. and Khokhlov, A.R. (2001) Two Types of Hydrophobic Aggregates in Aqueous Solutions of Chitosan and Its Hydrophobic Derivative. Biomacromolecules, 2, 483-490. http://dx.doi.org/10.1021/bm005649a 
Scientific Research Publishing (SCIRP) is one of the largest Open Access journal publishers. It is currently publishing more than 200 open access, online, peer-reviewed journals covering a wide range of academic disciplines. SCIRP serves the worldwide academic communities and contributes to the progress and application of science with its publication.

Other selected journals from SCIRP are listed as below. Submit your manuscript to us via either submit@scirp.org or Online Submission Portal.
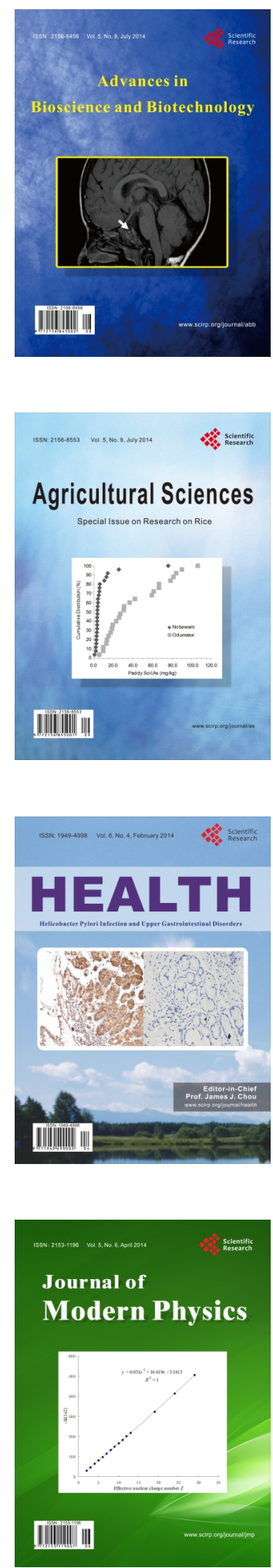
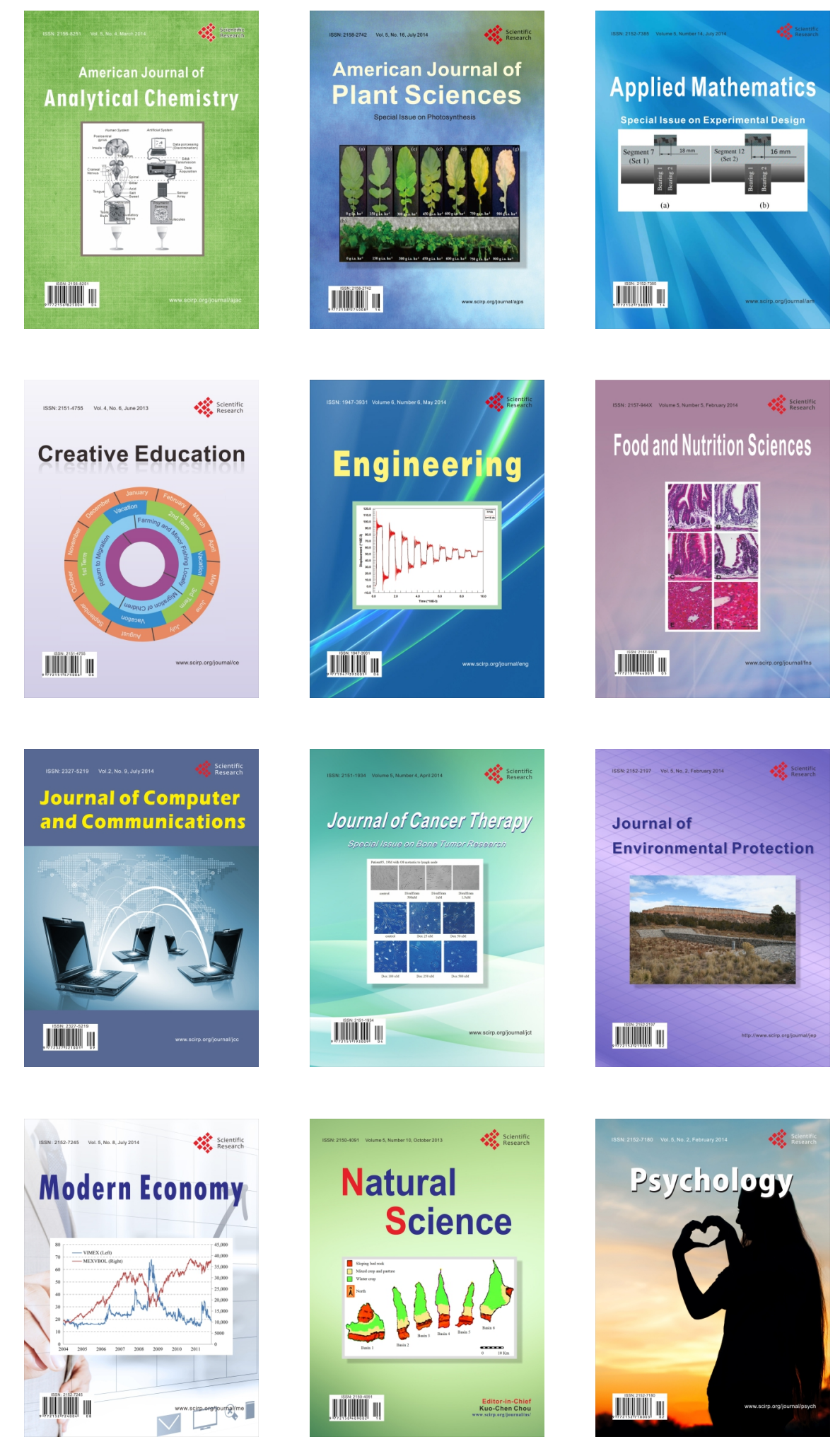